\title{
Relation between Renal Dysfunction and Liver Disease: A Retrospective Study
}

\author{
Siddarth Sadhnani \\ Sadhnani Medical Director, Siddhartha Endocrine Diagnostic Centre, 202, Moghal's Deccan Towers, Basheerbagh, \\ Hyderabad-32, India
}

Corresponding author: Siddhartha, Sadhnani Medical Director, Siddhartha Endocrine Diagnostic centre, 202, Moghal's Deccan Towers, Basheerbagh, Hyderabad-32, India

DOI: $10.21276 /$ ijcmsr.2018.3.3.33

How to cite this article: Siddarth Sadhnani. Relation between renal dysfunction and liver Disease: a retrospective study. International Journal of Contemporary Medicine Surgery and Radiology. 2018;3(3):C150-C152.

\section{A B S T R A C T}

Introduction: Chronic liver disease (Cirrhosis) is a progressive disease, develops slowly over many years. If it is untreated and allowed to continue, results in scar tissue formation and finally stoppage of liver function. When healthy liver tissue is replaced by scar tissue, blocking the flow of blood through the liver and the condition becomes serious. The common and serious problem that the patients with the chronic liver disease is Renal dysfunction. Patients with chronic liver disease are more prone to kidney disease than the normal individuals. Chronic liver disease or cirrhosis, is characterized by functional renal disturbance without much changes in renal histology. Cirrhosis was generally thought to be irreversible but recent studies have shown that treatments aimed at the underlying cause of the disease can improve or even reverse fibrosis. So care should be taken for the identification of renal dysfunction in chronic liver disease or liver cirrhosis patients. Hence; the present study was done to assess the relation of renal profile with the liver cirrhosis patients.

Material and methods: The present study included evaluation of liver cirrhosis patients and finding of correlation of severity of disease with the renal profile. A total of 50 liver cirrhosis patients were included in the present study. Demographic and clinical details of all the patients were obtained. Physical examination was done to look for any evidence of cardiac or renal involvement in each and every patient. All the results were analyzed by SPSS software.

Results: A total of 50 liver cirrhosis patients were included in the present study. Out of 50, 40 male patients, 10 female patients. Alcohol was the most common cause the other causes include liver infection, immune system abnormality, genetic etc.

Conclusion: Renal function is altered in liver cirrhosis patients or chronic liver disease patients.

Key Words: Renal Dysfunction, Liver Disease, Cirrhosis, Hepatorenal Syndrome

\section{INTRODUCTION}

Cirrhosis is a final outcome of chronic liver disease and characterized by fibrosis, scarring, and leading to distortion of the hepatic vasculature. ${ }^{1}$ Cirrhosis is a common complication of chronic liver disease. In chronic liver disease changes takes place ie, loss of liver cells and irreversible scarring of the liver. Alcohol was the most common cause the other causes include liver infection(viral hepatitis B and C), immune system abnormality, genetic etc. Symptoms of Cirrhosis include weakness, loss of appetite, easy bruising, yellowing of the skin (jaundice), itching, and fatigue. Diagnosis of cirrhosis by history, physical examination and blood tests, hepatic and renal investigations. In every patient physical examination was done to look for any evidence of cardiac or renal involvement. Cirrhosis is asymptomatic and unnoticed until complications of liver disease present. In some patients never come to know that they are suffering from liver disease, and undiagnosed liver disease is frequently found at autopsy. In majority of cases the diagnosis of asymptomatic cirrhosis is usually made accidentally when screening was done or radiologic findings suggest liver disease. The complications of cirrhosis include Gastrointestinal bleeding, Diabetes, hepatic encephalopathy, Systemic hypertension, hepatocellular carcinoma, hepatorenal syndrome, peritonitis, and coagulation disorders. Cirrhosis with renal dysfunction is most serious complication and associated with morbidity and mortality. ${ }^{2}$ Hence special care should be given for the recognition of the acute or chronic renal disease. The parameters of renal dysfunction are creatinine and blood urea nitrogen and the parameters of liver dysfunction (Child-Pugh score and its components) used to determine the prognosis of cirrhosis. ${ }^{3}$ Cirrhosis is accompanied by distortion of the hepatic vasculature, failing to remove toxins from the blood, accumulate in the brain. ${ }^{4}$. The toxins in the brain is called hepatic encephalopathy. In this mental function decreases, resulting in stupor and coma. ${ }^{5}$ Hence; the present study was done to assess the relation of renal profile with the liver cirrhosis patients.

\section{MATERIAL AND METHODS}

The study was conducted at Siddhartha Endocrine Diagnostic centre \& clinic Basheerbagh, Hyderabad, 
Telangana, over period of 5 months. The study included liver cirrhosis patients from out patient department of medicine and finding of relation between liver disease and renal profile. Informed consent was obtained after explaining the research procedure. Ethical Clearance was obtained from Institutional Ethics Committee

A total of 50 liver cirrhosis patients were included in the present study. Out of 50, 40 male patients, 10 female patients. Patients of acute liver disease, hepatocellular carcinoma/ any malignancy, chronic renal failure, rheumatic heart disease, collagen disease, Patients on any form of medicine and patients with incomplete data were not included in this study are excluded from the study. clinical details of all the patients were obtained. ultrasound abdomen and pelvis, chest $\mathrm{x}$-ray, and report of laboratory investigations like complete blood count, liver function test, blood urea, serum creatinine, urine analysis, ascetic fluid analysis, serum sodium, serum potassium and urinary sodium were collected. Physical examination was done to look for any evidence of cardiac or renal involvement in each and every patient.

\section{STATISTICAL ANALYSIS}

The obtained data was analyzed by SPSS software. Chisquare test and student $t$ test was used for assessment of level of significance. P- value of less than 0.05 was taken as significant.

\section{RESULTS}

A total of 50 liver cirrhosis patients were included in this study. The mean age of patients was $46.32 \pm 10.19$ years. Of these, majority were male 40 and females were 10 (Table 1). According to Child-Pugh's score Liver disease is classified which is mentioned below (Table 2 and Table 3 ).

The most common etiology of cirrhosis was alcohol $85 \%$, followed by Hepatitis B $11 \%$ and C virus 4\% (Table 4). Renal dysfunction was observed in $30.3 \%$ of cirrhotic cases with viral etiology. It was found that 32 normal and 18 raised Blood urea and similar in Serum creatinine levels in all 50 patients (Table 5). According to Child Pugh Score from all 50 subjects maximum 42 comes in B Grade, 4 each in $\mathrm{A}$ and $\mathrm{C}$ grade. Renal profile of the liver cirrhosis patients was significantly $\left(\mathrm{p}<0.007^{*}\right)$. Complications of cirrhosis such as diabetes, hypertension, hepatic encephalopathy and gastrointestinal bleeding.

Child-Pugh score and its components are used to determine the prognosis of cirrhosis.

It includes five clinical measures of liver disease. Each measure is scored $1-3$, with 3 points indicating most severe derangement.

Encephalopathy divided into absent 1point, grade 1: Altered mood/confusion (2 points),In appropriate behavior, impending stupor,somnolence(2points), grade 3 markedly confused, stuporous but arousable (3 points), grade 4 Comatose/unresponsive ( 3 points)

Chronic liver disease is classified into Child-Pugh class A to $\mathrm{C}, \mathrm{A}-(5-6), \mathrm{B}(7-9) \mathrm{C}(10-15), \mathrm{C}$ indicating most severe derangement.

Renal parameters (Blood urea and serum creatinine)

\begin{tabular}{|l|c|}
\hline Gender & No. of Patients \\
\hline Males & 40 \\
\hline Females & 10 \\
\hline Total & 50 \\
\hline \multicolumn{2}{|c|}{ Table-1: Distribution of subjects according to gender } \\
\hline
\end{tabular}

\begin{tabular}{|l|c|c|}
\hline Measure & Values & Grading \\
\hline Total Bilirubin & $<2$ & 1 point \\
\hline & $2-3$ & 2 points \\
\hline Serum Albumin & $>3$ & 3 points \\
\hline & $>3.5$ & 1 point \\
\hline & $2.8-3.5$ & 2 points \\
\hline Prothrombin time & $<2.8$ & 3 points \\
\hline & $<4$ & 1 point \\
\hline & $4-6$ & 2 points \\
\hline Ascites & $>6$ & 3 points \\
\hline & Absent & 1 point \\
\hline & Mild & 2 points \\
\hline Hepatic Encephalopathy & Moderate-Severe & 3 points \\
\hline \multicolumn{2}{|c|}{ Absent } & 1 point \\
\hline & Grade 1-2 & 2 points \\
\hline \multicolumn{2}{|c|}{ Grade 3-4 } & 3 points \\
\hline \multicolumn{2}{|c}{} \\
\hline
\end{tabular}

\begin{tabular}{|l|c|c|}
\hline Child-Pugh score & Points & No. of Subjects \\
\hline A & $5-6$ & 4 \\
\hline B & $7-9$ & 42 \\
\hline C & $10-15$ & 4 \\
\hline \multicolumn{2}{|c|}{ Table-3: Child-Pugh class A to C grading and distribution of } \\
subjects \\
\hline
\end{tabular}

\begin{tabular}{|l|c|}
\hline Etiology & No. of Patients \\
\hline Alcohol & $85 \%$ \\
\hline Hepatitis B & $11 \%$ \\
\hline Hepatitis C & $4 \%$ \\
\hline \multicolumn{2}{|c|}{ Table-4: Etiology of Cirrhosis } \\
\hline
\end{tabular}

reflects glomerular filtration rate(GFR). In healthy kidneys glomerular filtration rate with in the normal limits $(125 \mathrm{ml} /$ min). As kidney function decreases the excretion of Blood urea and serum creatinine decreases with increases of both in blood concentration. Normal levels of blood urea-2.5$7.1 \mathrm{mmol} / \mathrm{L}$,Normal serum creatinine levels $88-128 \mathrm{ML} /$ min in women, $97-137$ in male. In our study we observed raised urea and creatinine levels in cirrhotic patients. Renal function is measured by blood urea, and serum creatinine levels. In renal disease decrease in GFR leads to increase in renal parameters mostly serum creatinine, when it exceeds normal levels changes in GFR takes place. Persistent increase in serum creatinine levels results in development of end stage of renal disease.

\section{DISCUSSION}

Most common etiology of cirrhosis $(n=50)$ in our study was alcoholism (85\%) followed by hepatitis B(11\%) and Hepatitis C infection (4.\%). According to Kumar T. alcoholism the 
most common cause of cirrhosis of liver. ${ }^{6}$ However, Joshi et al. Chronic hepatitis ' $\mathrm{B}$ ' is the common cause of cirrhosis of liver. ${ }^{7}$ The disparity between these studies explained by The National Family Health Survey that the higher prevalence of alcohol consumption more the damage of liver and common among males in India.

In advance stages of chronic liver disease, results in circulatory stress promotes heart failure which accentuates arterial under filling with decreased renal perfusion resulting in deterioration of renal function. ${ }^{8}$ In the present study, we observed that renal manifestation was associated with liver cirrhosis patients. Chronic Liver disease is a major health problem. Renal failure in cirrhosis is one of the most important risk factors when liver transplantation is being considered. ${ }^{9}$ Cirrhotic patients with renal failure are at high risk for mortality. Liver transplantation in cirrhotic patients with renal failure have an increased incidence of complications with reduced survival after transplantation. Renal dysfunction has occurred in hospitalized patients with cirrhosis and the most common cause is AKI, It has been documented AKI $(22 \%)$ occur mostly secondary to hypovolemia (gastrointestinal haemorrhage, aggressive diuresis, or diarrhoea), infection and drugs. ${ }^{10} \mathrm{Chronic}$ kidney injury appears to be a rare diagnosis as seen in $3 \%$ of the cirrhotic patients. ${ }^{11}$ Hepatorenalsyndrome constitutes $31.8 \%$ of the cases with renal dysfunction and, it accounts for $7 \%$ of cases of hospitalized cirrhotic patients. Hepatorenalsyndrome (HRS) was described by Hecker and Sherlock. ${ }^{12}$ Chayanupatkul $\mathrm{M}$ et al explained that the pathophysiology of cirrhotic cardiomyopathy is characterized by the impaired systolic response to physical stress, diastolic dysfunction as well as electrophysiological abnormalities especially prolongation of QT interval. ${ }^{13}$ According to Peter $\mathrm{G}$ et al prolongation of QT interval is an risk factor for development of hepatorenal syndrome in cirrhotic patients and found that hepatorenal syndrome developed in $15.9 \%$ patients. ${ }^{14}$

\section{CONCLUSION}

From above study, special care should be taken in treating chronic liver disease patients with regard to their renal function. Hence early diagnosis, prevention and treatment of renal dysfunction can reduce the morbidity and mortality.

\section{ACKNOWLEDGMENTS}

We express our sincere gratitude to patents and staff for their constantsupport to carry over this study.

\section{REFERENCES}

1. Sharma M, Rameshbabu CS. Collateral Pathways in Portal Hypertension. Journal of Clinical and Experimental Hepatology. 2012;2(4):338-52.

2. Pipili C, Cholongitas E. Renal dysfunction in patients with cirrhosis: Where do we stand? World J GastrointestPharmacolTher2014; 5(3):156-68.

3. D'Amico G, Garcia-Tsao G, Pagliaro L. Natural history and prognostic indicators of survival in cirrhosis: a systematic review of 118 studies. J Hepatol 2006; 44(5):217-31.

4. Massimo Pinzani et al. Liver cirrhosis.Best Practice and Research Clinical Gastroenterology. 2011; 25(2): 281-290.

5. Reichen J, Egger B, Ohara N, Zeltner TB, Zysset T, Zimmermann A. Determinants of hepatic function in liver cirrhosis in therat. Multivariate analysis. J Clin Invest 1988;82(3):2069-76.

6. Kumar T. Incidence of cirrhosis caused by hepatitis $\mathrm{b}$ virus in different sex and age groups in Bihar. J MGIMS. 2006;11(1):52-54.

7. Joshi PH. Chronic hepatitis 'B'. Bombay Hosp J. 1996;3804(712):701

8. Cholongitas, E; Papatheodoridis, GV; Vangeli, M; Terreni, N; Patch, D; Burroughs, AK. Systematic review: The model for end-stage liver disease--should it replace Child-Pugh's classification for assessing prognosis in cirrhosis?. Alimentary pharmacology and therapeutics. 22 (11-12): 1079-89.

9. Gonwa TA, Klintmalm GB, Levy M, Jennings LS, Goldstein RM, Husberg BS. Impact of pretransplant renal Jaiganesh Mohan et al / Renal dysfunction in cirrhotic liver 76 IJBR (2016) 7(02) www.ssjournals. com function on survival after liver transplantation. Transplantation 1995; 59:361-5.

10. Woitas RP, Heller J, Stoffel-Wagner B, Spengler U, SauerbruchT. Renal functional reserve and nitric oxide in patients with compensatedliver cirrhosis. Hepatology 1997;26(6):858-64.

11. Prakash J, Mahapatra AK, Ghosh B, Arora P, Jain AK. Clinical spectrum of renal disorders in patients with cirrhosis of liver. Ren Fail 2011; 33(5):40-6.

12. Hecker R, Sherlock S. Electrolyte and circulatory changes in terminal liver failure. Lancet. 1956;2(3):12211225

13. Chayanupatkul M, Liangpunsakul S. Cirrhotic cardiomyopathy: review of pathophysiology and treatment. Hepatology international. 2014;8(3):308315.

14. Peter G, George PC, Villyoth MP, Sivaraman S, Hamza RE et al. QT interval prolongation: a risk factor for development of hepatorenal syndrome in cirrhotic patients with acute variceal bleeding. QT interval prolongation: a risk factor for development of hepatorenal syndrome in cirrhotic patients with acute variceal bleeding. Tropical Gastroenterology 2014;35(3):157-163.

\section{Source of Support: Nil; Conflict of Interest: None}

Submitted: 03-08-2018; Accepted: 20-09-2018; Published online: 27-09-2018 